\title{
Relaxation Dynamics and Genuine Properties of the Solvated Electron in Neutral Water Clusters
}

\section{Journal Article}

\section{Author(s):}

Gartmann, Thomas E.; Ban, Loren (D); Yoder, Bruce L.; Hartweg, Sebastian; Chasovskikh, Egor; Signorell, Ruth (D)

Publication date:

2019-09

Permanent link:

https://doi.org/10.3929/ethz-b-000362750

Rights / license:

In Copyright - Non-Commercial Use Permitted

Originally published in:

The Journal of Physical Chemistry Letters 10(17), https://doi.org/10.1021/acs.jpclett.9b01802

\section{Funding acknowledgement:}

786636 - Droplet Photoelectron Imaging (EC)

125760 - Molecular Ultrafast Science and Technology (MUST) (SNF)

172472 - Phase Transitions of Ultrafine Aerosol Particles: Condensation, Freezing, and Metal Formation in Confined Systems (SNF) 


\title{
Relaxation Dynamics and Genuine Properties of the Solvated Electron in Neutral Water Clusters
}

\author{
Thomas E. Gartmann, ${ }^{\dagger}$ Loren Ban, ${ }^{\dagger}$ Bruce L. Yoder, Sebastian Hartweg, Egor Chasovskikh, \\ and Ruth Signorell*i)
}

Department of Chemistry and Applied Biosciences, Laboratory of Physical Chemistry, ETH Zürich, Vladimir-Prelog-Weg 2, CH-8093 Zürich, Switzerland

\section{Supporting Information}

ABSTRACT: We have investigated the solvation dynamics and the genuine binding energy and photoemission anisotropy of the solvated electron in neutral water clusters with a combination of time-resolved photoelectron velocity map imaging and electron scattering simulations. The dynamics was probed with a UV probe pulse following above-band-gap excitation by an EUV pump pulse. The solvation dynamics is completed within about 2 ps. Only a single band is observed in the spectra, with no indication for isomers with distinct binding energies. Data analysis with an electron scattering model reveals a genuine binding energy in the range of 3.55-3.85 eV and a genuine anisotropy parameter in the range of $0.51-0.66$ for the ground-state hydrated electron. All of these observations coincide with those for liquid bulk, which is rather unexpected for an average cluster size of 300 molecules.

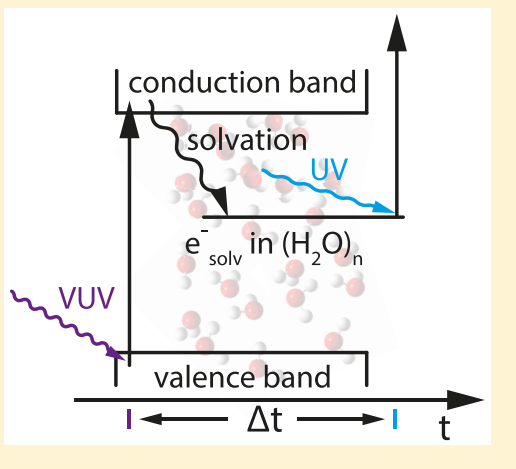

$\mathrm{T}$ he broad attention that solvated electrons in their ground and excited states have attracted over many decades can be attributed to them being among the simplest quantum solutes as well as to their important role in a wide range of fields. Many studies have focused on excess electrons in water and anion water clusters ${ }^{1-37}$ (and refs therein). For liquid water, the excited-state relaxation dynamics of electrons has been investigated over a broad time window from femtoseconds to beyond picoseconds. This has resulted in the picture of an initially delocalized electron that relaxes to a hydrated electron within $\sim 1$ ps; subsequent geminate recombination takes place on a much longer time scale ${ }^{11,16}$ (and refs therein). Typically, multiphoton ionization of neat water using different detection schemes has been employed in these investigations. Recent time-resolved photoelectron studies have revealed a nonadiabatic transition from excited electronic states ( $\mathrm{p}$-states) to the ground electronic state ( $\mathrm{s}$ state) of the hydrated electron on a sub-100 fs time scale followed by slow (several $100 \mathrm{fs}$ ) relaxation in the ground electronic state ${ }^{9,10,38}$ (and refs therein). Analogous studies of the relaxation dynamics in clusters-often after an $s$ to $p$ excitation-were conducted for different cluster sizes in photodetachment (anionic cluster) experiments ${ }^{12,14,15,31,35}$ (and refs therein) and in neutral clusters after excitation by an extreme ultraviolet (EUV) light pulse. ${ }^{39}$

For the electronic ground state (s), several binding motifs (isomers) have been identified in anionic clusters including fully and partially solvated internal states and surface-bound states (refs 12, 21, 28, 40, and references therein). Distinct experimental vertical binding energies (VBEs) have been observed for the different isomers, with a strong cluster size dependence. The difference in VBE between an internal and surface state amounts to about $1 \mathrm{eV}$ for a cluster of 200 molecules. Extrapolation of the cluster data to the infinite bulk results in binding energies of 3.6 and $1.6 \mathrm{eV}$ for the fully solvated internal and surface-bound state, respectively, which lie 1.3 and $0.3 \mathrm{eV}$, respectively, below the value for a cluster with 200 molecules. Most experimental studies of the liquid bulk (liquid microjets) find only a single band in the range of 3.3-3.6 eV (refs 2, 8, 37, and references therein). The only exception is an additional surface-bound state at $1.6 \mathrm{eV}$ reported in ref 5 , which has not been reproduced so far by other studies, although a recent EUV study on neutral clusters again speculates about such as surface state. ${ }^{39}$ A large number of overlapping bands from different species in this cluster spectrum makes an assignment to different contributions questionable.

In order to characterize the dynamics and different states of the hydrated electron, photoelectron studies record the photoelectron kinetic energies (eKEs) and in some cases the photoelectron angular distributions (PADs) usually characterized by a single anisotropy parameter $\beta$. Our recent investigation on hydrated electrons in liquid water microjets has, however, revealed that these measurement quantities are strongly influenced by electron transport scattering in the liquid and thus depend on the photon energy of the ionizing light source. ${ }^{37}$ Typically, experimental VBEs vary by about 1 $\mathrm{eV}$ depending on the energy of the ionizing photon. Furthermore, the experimental PADs of the hydrated electron

Received: June 21, 2019

Accepted: August 5, 2019

Published: August 5, 2019 
in the liquid correspond to an almost isotropic distribution because of electron transport scattering. Proper analysis of the data thus requires the influence of electron scattering to be taken into account. ${ }^{37,41-50}$ We have shown that corrections of the experimental data by means of a detailed electron scattering model make it possible to retrieve a genuine (intrinsic) VBE and $\beta$ for the ground state hydrated electron of $3.7 \pm 0.1 \mathrm{eV}$ and $0.6 \pm 0.2$, respectively. ${ }^{37,41,42}$ In large clusters, the electron scattering is substantially different from that of the bulk (liquid/amorphous solid), as shown in our recent study. ${ }^{51}$ While electron scattering cross sections in the liquid and the amorphous solid are virtually identical within experimental uncertainties, those for clusters are significantly larger and lie between the gas- $^{52}$ and condensed-phase values. ${ }^{37,41,42}$ The reduced dielectric screening in clusters compared with that in the condensed phase provides a simple explanation for the increased scattering cross sections in the cluster. ${ }^{51}$

The present study investigates the relaxation dynamics in large water clusters with $\sim 300$ molecules after above-band-gap excitation by an EUV photon from a high harmonic laser source (pump) and ionization by a UV probe pulse and the properties of the resulting ground-state solvated electron. It has so far been unknown how the behavior of the solvated electron in large neutral clusters differs from that in large anionic clusters and in liquid bulk. The role that the environment has on the properties of the solvated electron is of general interest, especially regarding confined environments and interfaces. ${ }^{21}$ By analogy to anionic clusters, one would expect significant polarization effects shifting the VBEs of clusters relative to the bulk and possibly also isomers with different VBEs. Surprisingly, this is not what we find. By properly accounting for the effects of electron scattering in the clusters, we have evaluated genuine cluster VBEs and $\beta$ parameters, which can be directly compared with the corresponding bulk values.

The experimental setup used for the measurements in the present work has been described previously. ${ }^{51,53}$ We used a velocity map imaging $(\mathrm{VMI})^{54}$ spectrometer $^{55-58}$ to record time-resolved photoelectron images upon photoionization of water clusters using a pump and probe laser excitation scheme. EUV light was produced by high harmonic generation (HHG), ${ }^{59}$ as previously described. ${ }^{51}$ A home-built, timepreserving monochromator ${ }^{60,61}$ was used to select the seventh harmonic $(\sim 10.9 \mathrm{eV})$, which was used as the pump. As a probe pulse, we used $266 \mathrm{~nm}$ light generated by frequency tripling of the $800 \mathrm{~nm}$ output from a Ti:sapphire laser. A beam of neutral water clusters was produced and characterized in the same manner as in previous work. ${ }^{51}$ For the experiments presented in this work, the average cluster size was determined to be $\langle n\rangle$ $=300$ via the sodium-doping method. ${ }^{62,63}$ See section S.1 in the Supporting Information (SI) for details.

The electron scattering simulations have been described previously in detail. ${ }^{37,41,42,50,51}$ The probabilistic scattering model is based on a Monte Carlo solution of the transport equation. The scattering cross sections, angular dependences, and energy loss functions of all relevant elastic and inelastic phonon, vibron, and electronic scattering channels are explicitly taken into account. Simulations are performed for cluster scattering cross sections (models iii and iv), ${ }^{51}$ liquid water scattering cross sections (model i), ${ }^{51}$ and gas-phase scattering cross sections (model ii). ${ }^{51}$ While the scattering cross sections used in models $i$ and iii are taken directly from the previous study, ${ }^{51}$ the cross sections in models ii and iv were adapted to account for the lower electron kinetic energies in the current study. See section S.2 in the SI for details.

Figure 1 shows the time evolution of the vertical electron binding energy (VBE, panel a), and the full width at half-

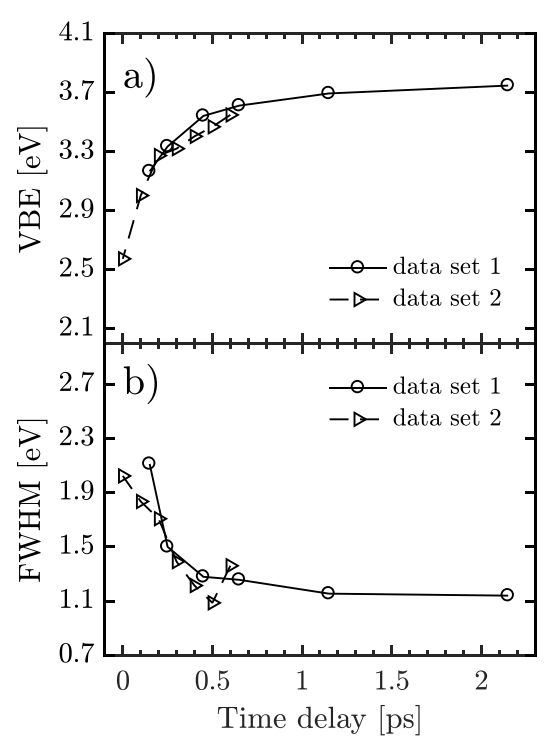

Figure 1. Evolution of the photoelectron signal as a function of the pump-probe time delay. (a) Vertical electron binding energy (VBE) and (b) full width at half-maximum (fwhm). Uncertainties are estimated not to exceed \pm 0.2 and $\pm 0.3 \mathrm{eV}$ for the VBE and fwhm, respectively. Lines connecting data points are intended as a guide to the eye. Data sets 1 and 2 represent independent measurement series, providing an impression of the reproducibility of the experiment.

maximum (fwhm, panel b), of the binding energy spectra recorded after above-band-gap excitation with EUV light of $\sim 10.9 \mathrm{eV}$ photon energy. The VBE increases with time and stabilizes after $1-2 \mathrm{ps}$ at a value of $\sim 3.75 \mathrm{eV}$, while the fwhm narrows on the same time scale to stabilize at $\sim 1.14 \mathrm{eV}$ (see section S.3 in the SI with binding energy spectra and fits at different pump-probe delays in Figure S3). The temporal increase in the VBE reflects the formation of a more strongly bound hydrated electron over time. The fast increase of the VBE by about $1 \mathrm{eV}$ in the first few hundred fs cannot be fully resolved in our experiment with a pump-probe cross correlation of about $220 \mathrm{fs}$ (fwhm). We assume that after 2 ps a hydrated electron in its electronic ground (s) state has formed in an equilibrated solvent environment. Direct comparison with previous studies is difficult because the time scales for equilibration depend strongly on how the electron was initially prepared. ${ }^{32}$ Still, the overall solvation time scale of a few ps is in reasonable agreement with previously reported time scales for the formation of the ground-state hydrated electron in liquid bulk water and in anionic water clusters $^{12,16,23,31,32,35,36,38}$ (and refs therein). Considering that initial ejection lengths for excitation at $\sim 10.9 \mathrm{eV}$ in the liquid are $\sim 3 \mathrm{~nm},{ }^{11}$ which exceeds the cluster radius of $\sim 1.3 \mathrm{~nm}$, it is plausible that most of the initial electron density resides near the surface. This situation closely resembles the model calculation of Herbert and co-workers for the localization of surface electrons and their internalization into the bulk. ${ }^{17}$ Comparing the calculated time evolution of the VBE with our experimental results in Figure 1 and accounting for the cross correlation of our experiment, we find reasonable agreement with a major change of the VBE in the first $0.5 \mathrm{ps}$. The initial $\beta$ 
parameter $(\sim 0.4)$ that we measure exceeds that of the electronic ground state $(\sim 0.19)$. This might indicate an initially larger $\mathrm{s}$ character or less electron scattering upon ionization. The latter does not appear unreasonable for an electron residing close to the surface.

By combining experimental photoelectron VMI images with detailed scattering simulations, we have recently determined genuine vertical binding energies and genuine $\beta$ parameters for the ground-state hydrated electron in liquid bulk water. ${ }^{37}$ Here, we retrieve genuine VBEs (Figure 2) and genuine $\beta$

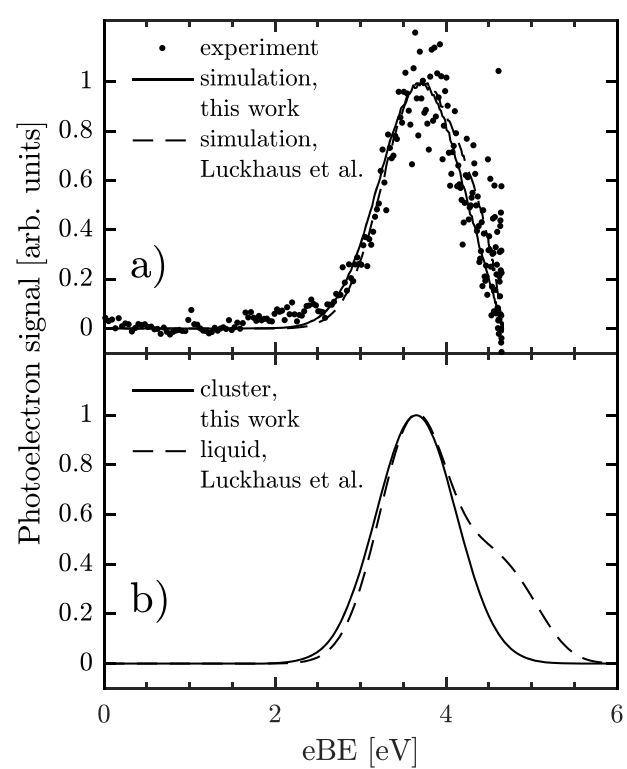

Figure 2. (a) Experimental binding energy spectrum recorded at a pump-probe delay of $\sim 2$ ps (circles). Dashed line spectrum: Simulation with genuine eBE of the liquid from Luckhaus et al. ${ }^{37}$ (dashed line in panel b). Full line spectrum: Simulation with a single Gaussian fit for the genuine cluster eBE (full line in panel $b$ ).

parameters (Figure 3) for the ground-state hydrated electron in large neutral water clusters from the photoelectron VMI images of the clusters recorded after $\sim 2$ ps (Figure 1). Figure 2 a shows the experimental binding energy spectrum at $\sim 2$ ps time delay together with two simulated spectra. For the

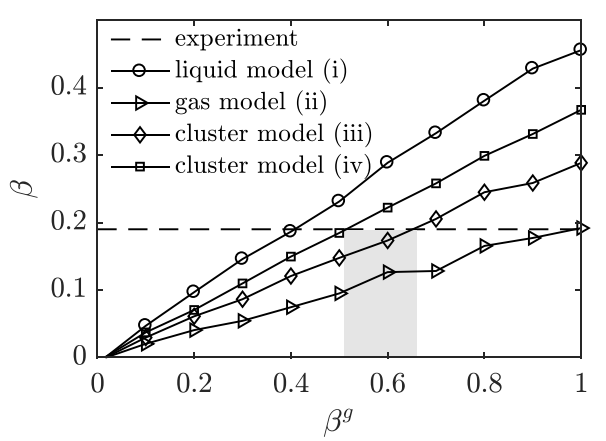

Figure 3. Observable $\beta$ values as a function of genuine $\beta$ values $\left(\beta^{\mathrm{g}}\right)$ simulated for different models of the electron scattering cross sections (average cluster size $\langle\mathrm{n}\rangle=300$; see ref 51 for simulation details). Lines in between data points represent linear interpolations. The horizontal dashed line represents the experimental $\beta$ value of 0.19 . The gray shaded area indicates the range of genuine $\beta$ parameters bracketed by cluster models iii and iv, which is consistent with the experimental $\beta$ value measured for water clusters. calculated, dashed line spectrum, we used the genuine binding energy spectrum of the liquid (see the starred spectrum in Figure 3 of Luckhaus et al..$^{37}$ and the dashed line spectrum in Figure $2 \mathrm{~b}$ ) and the cluster scattering cross sections (model iii from ref 51) for the scattering calculations. The calculated, solid line spectrum was obtained by fitting a genuine binding energy spectrum (solid line in Figure $2 b$ ) to the experimental data using scattering calculations with the same cluster scattering cross sections (model iii in ref 51). Contrary to the liquid bulk experiments, ${ }^{37}$ the experimental cluster data (Figure 2a) were obtained for a single probe energy $(266 \mathrm{~nm}$ ), which is too low to cover the shoulder in the genuine binding energy spectrum of the liquid bulk (dashed line spectrum in Figure 2b). A single Gaussian is thus sufficient to represent the main part of the genuine binding energy spectrum of the clusters (full line in Figure 2b) covered by the experiment. Both simulations in Figure $2 \mathrm{a}$ represent the experimental spectrum very closely, suggesting that the genuine binding energy spectra of the hydrated electron in the liquid bulk and in a neutral water cluster with $\sim 300$ molecules are similar. From the scattering correction including a sensitivity analysis using different scattering cross sections for clusters (models iii and iv from ref 51), we derived a genuine VBE of 3.55-3.85 $\mathrm{eV}$ for the hydrated electron in neutral clusters (Table 1). This

Table 1. Values for the Genuine VBE and Genuine $\beta$ Parameter for the Solvated Electron in Liquid Water in the Bulk and at the Air/Water Interface Region, in Neutral Water Clusters, and in Anionic Water Clusters

\begin{tabular}{|c|c|c|}
\hline & $\begin{array}{c}\text { genuine VBE } \\
{[\mathrm{eV}]}\end{array}$ & $\begin{array}{l}\text { genuine } \beta \\
\text { parameter }\end{array}$ \\
\hline calc. bulk from refs 17 and 21 & $3.4-3.6$ & \\
\hline calc. bulk from ref 64 & $3.75 \pm 0.55^{a}$ & \\
\hline calc. interfacial from refs 17 and 21 & $3.1-3.2$ & \\
\hline calc. interfacial from ref 64 & $3.35 \pm 0.46^{a}$ & \\
\hline exp. liquid bulk ${ }^{37}$ & $3.7 \pm 0.1$ & $0.6 \pm 0.2$ \\
\hline $\begin{array}{l}\text { exp. neutral cluster ( } 300 \text { molecules); this } \\
\text { work }\end{array}$ & $3.55-3.85$ & $0.51-0.66$ \\
\hline exp. anion cluster ${ }^{14}$ ( $\sim 50$ molecules $)$ & & $\sim 0.7 \pm 0.1$ \\
\hline
\end{tabular}

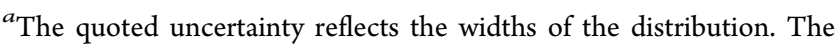
uncertainty in the peak positions is probably on the order of \pm 0.1 .

coincides surprisingly well with the experimental liquid value of $3.7 \pm 0.1 \mathrm{eV}$. As most of the liquid bulk studies, we do not find any evidence for a surface-bound electron with a VBE at $1.6 \mathrm{eV}$ (Figures 2 and S3). Contrary to the anionic clusters, the neutral clusters show only a single VBE band and, even more surprisingly, no significant shift relative to the bulk, which one would have expected from polarization effects.

As a result of electron scattering, the PAD measured for bulk liquid water is almost isotropic ( $\beta$ close to 0$).{ }^{1}$ Properly accounting for the scattering contributions, this leads to a genuine $\beta$ parameter in the liquid bulk of $0.6 \pm 0.2 .^{37}$ The value seems in fair agreement with what one would expect for an s-like orbital character of the ground-state solvated electron. ${ }^{21}$ For clusters with $\sim 300$ molecules, we measure a $\beta$ parameter of $0.19 \pm 0.12$ at a $\sim 2$ ps time delay (dashed horizontal line in Figure 3). (Absolute values for $\beta$ parameters are typically not measured to better than \pm 0.1 accuracy with VMI setups.) The measured value is again strongly reduced compared with the genuine $\beta$ parameter in the cluster due to electron scattering processes. To retrieve the genuine $\beta$ 
parameter in the clusters, we performed scattering simulations, with the results shown in Figure 3. The diamonds and the squares show the expected measured $\beta$ parameter as a function of the genuine $\beta$ parameter for scattering simulations with the cluster cross sections from models iii and iv, respectively. According to the graph, the measured $\beta$ parameter of 0.19 for the clusters is consistent with a genuine $\beta$ parameter in the range of $0.51-0.66$, depending on the scattering cross sections used for the clusters. ${ }^{51}$ As the VBE, the genuine $\beta$ parameter of the cluster lies remarkably close to the genuine value of the liquid bulk (Table 1 ) and to the value of $\sim 0.7 \pm 0.1$ reported for anionic water clusters containing $\sim 50$ molecules. ${ }^{14}$ The simulations in Figure S5 in the SI provide an idea of the cluster size dependence of the measured $\beta$ parameter predicted as a function of the genuine $\beta$ parameter for clusters with $100-900$ molecules.

Figure 3 also shows results for scattering simulations where we have replaced the scattering cross sections of the clusters, either by the scattering cross section of the liquid phase ${ }^{37,41,42}$ (circles) or by those of the gas phase ${ }^{52}$ (triangles). For liquid scattering cross sections, our experimental $\beta$ parameter of 0.19 would lead to a genuine $\beta$ parameter of $\sim 0.4$, while for the gasphase scattering cross sections a genuine $\beta$ parameter of $\sim 1.0$ would result. Neither would be fully consistent with the results of the liquid bulk. This further underlines the importance of using proper cross sections to account for electron transport in a cluster.

It is rather unexpected to find the same properties for the solvated electron in large neutral clusters as have been found in the liquid bulk, without any need for extrapolation to infinite cluster size. How could this be rationalized? As is the case for most cluster studies, we do not have direct information regarding the temperature and phase of the cluster. For our type of supersonic expansion, we know that we produce warm clusters. It is thus conceivable that we produce liquid-like clusters, i.e., with relatively high mobility of solvent molecules. This is supported by our previous study of the single-photon EUV ionization of pure water clusters generated under precisely the same conditions, ${ }^{51}$ with ionization energies extrapolating to the liquid bulk value rather than that of the solid (see Figure 3 in ref 51). The high temperature of our clusters might explain why we do not observe the surfacesolvated electron. This is consistent with depletion of the corresponding signal for anionic clusters in warmer supersonic expansions. ${ }^{12,28,40}$ However, the fact that the eBE spectrum both in liquid bulk and in neutral clusters shows only one band does not prove the absence of multiple isomers. It only shows that there are no isomers with significantly different VBEs. Recent calculations by Herbert and co-workers ${ }^{17,21,64}$ have revealed similar VBE values for electrons solvated in the liquid bulk and at the air-liquid interface region (Table 1). All this still does not explain why the genuine VBE of the liquid bulk is already reached in neutral clusters with 300 molecules without further extrapolation to infinite cluster size, while the VBE of anionic clusters of similar size still lies $1 \mathrm{eV}$ below the bulk limit. Similar to our neutral water clusters, the bulk limit of the VBE of the solvated electron in neutral $\mathrm{Na}$-doped water clusters is already reached at very small cluster sizes. ${ }^{65,66}$ Both cases feature a charge-separated neutral ground state of the solvated electron and its counterion $\left(\mathrm{H}^{+}(\mathrm{aq})\right.$ in our case $)$. The presence of the counterion could explain why the bulk limit is already reached in our neutral clusters, provided that the charge-separated ground state experiences the same polarization shift as the cationic state resulting after ionization.

In conclusion, we have shown that formation of the groundstate hydrated electron in neutral water clusters with about 300 molecules after above-band-gap excitation is completed within about 2 ps. This is roughly consistent with the time scale of the solvation dynamics found in the liquid bulk and in large anion clusters, hinting at similar mechanisms in the different systems. Both the genuine VBE and the genuine $\beta$ parameter of hydrated electrons in large neutral clusters virtually coincide with the corresponding liquid bulk values, with only a single band in the spectrum. Contrary to anionic clusters, neither the bulk liquid nor the neutral clusters seem to give rise to different isomers with distinct VBEs. In particular, we do not find evidence for a surface-bound electron with a VBE of around $1.6 \mathrm{eV}$. That the liquid bulk value of the VBE is already reached for clusters containing about 300 molecules is rather surprising and indicates significant polarization effects in the charge-separated neutral ground state. Overall, our results suggest that the nature of the hydrated electron in neutral clusters is similar to that in the liquid bulk. Conclusive proof of this similarity, however, is not possible on the basis of VBEs and $\beta$ parameters alone. It will require further investigations with other measurement methods probing different (genuine) properties and will also need the support of theoretical modeling.

\section{ASSOCIATED CONTENT}

\section{S Supporting Information}

The Supporting Information is available free of charge on the ACS Publications website at DOI: 10.1021/acs.jpclett.9b01802.

Size determination of the neutral cluster distribution, typical mass spectrum for cluster size determination, corresponding neutral cluster size distribution, determination of electron scattering cross sections for clusters, total electron scattering cross sections for quasi-elastic and vibrational scattering in liquid water, gas-phase water, and clusters, VMI data analysis, time-dependent photoelectron spectra and corresponding Gaussian fits used to determine VBEs and fwhms, data for delays, size dependence of the VBE and the $\beta$ parameter, size dependence of simulated photoelectron spectra, and size dependence of simulated $\beta$ parameters for a range of genuine $\beta$ parameters (PDF)

\section{AUTHOR INFORMATION}

\section{Corresponding Author}

*E-mail: rsignorell@ethz.ch.

ORCID

Ruth Signorell: 0000-0003-1111-9261

\section{Author Contributions}

${ }^{\dagger}$ T.E.G. and L.B. contributed equally to this work.

\section{Notes}

The authors declare no competing financial interest.

\section{ACKNOWLEDGMENTS}

We thank David Stapfer and Markus Steger for technical support. This project received funding from the European Union's Horizon 2020 research and innovation program from the European Research Council under Grant Agreement No. 
786636, and the research was supported by the NCCR MUST, funded by the Swiss National Science Foundation (SNSF), through ETH-FAST and through SNSF Project No. 200020_172472.

\section{REFERENCES}

(1) Yamamoto, Y.-I.; Suzuki, Y.-I.; Tomasello, G.; Horio, T.; Karashima, S.; Mitríc, R.; Suzuki, T. Time- and Angle-Resolved Photoemission Spectroscopy of Hydrated Electrons near a Liquid Water Surface. Phys. Rev. Lett. 2014, 112, 187603.

(2) Yamamoto, Y.; Karashima, S.; Adachi, S.; Suzuki, T. Wavelength Dependence of UV Photoemission from Solvated Electrons in Bulk Water, Methanol, and Ethanol. J. Phys. Chem. A 2016, 120, 11531159.

(3) Horio, T.; Shen, H.; Adachi, S.; Suzuki, T. Photoelectron Spectra of Solvated Electrons in Bulk Water, Methanol, and Ethanol. Chem. Phys. Lett. 2012, 535, 12-16.

(4) Faubel, M.; Siefermann, K. R.; Liu, Y.; Abel, B. Ultrafast Soft XRay Photoelectron Spectroscopy at Liquid Water Microjets. Acc. Chem. Res. 2012, 45, 120-130.

(5) Siefermann, K. R.; Liu, Y.; Lugovoy, E.; Link, O.; Faubel, M.; Buck, U.; Winter, B.; Abel, B. Binding Energies, Lifetimes and Implications of Bulk and Interface Solvated Electrons in Water. Nat. Chem. 2010, 2, 274-279.

(6) Lübcke, A.; Buchner, F.; Heine, N.; Hertel, I. V.; Schultz, T. Time-Resolved Photoelectron Spectroscopy of Solvated Electrons in Aqueous NaI Solution. Phys. Chem. Chem. Phys. 2010, 12, 1462914634.

(7) Buchner, F.; Schultz, T.; Lübcke, A. Solvated Electrons at the Water-Air Interface: Surface Versus Bulk Signal in Low Kinetic Energy Photoelectron Spectroscopy. Phys. Chem. Chem. Phys. 2012, $14,5837-5842$.

(8) Shreve, A. T.; Elkins, M. H.; Neumark, D. M. Photoelectron Spectroscopy of Solvated Electrons in Alcohol and Acetonitrile Microjets. Chem. Sci. 2013, 4, 1633-1639.

(9) Elkins, M. H.; Williams, H. L.; Shreve, A. T.; Neumark, D. M. Relaxation Mechanism of the Hydrated Electron. Science 2013, 342, 1496-1499.

(10) Karashima, S.; Yamamoto, Y.; Suzuki, T. Resolving Nonadiabatic Dynamics of Hydrated Electrons Using Ultrafast Photoemission Anisotropy. Phys. Rev. Lett. 2016, 116, 137601.

(11) Elles, C. G.; Jailaubekov, A. E.; Crowell, R. A.; Bradforth, S. E. Excitation-Energy Dependence of the Mechanism for Two-Photon Ionization of Liquid $\mathrm{H}_{2} \mathrm{O}$ and $\mathrm{D}_{2} \mathrm{O}$ from 8.3 to $12.4 \mathrm{eV}$. J. Chem. Phys. 2006, 125, No. 044515.

(12) Young, R. M.; Neumark, D. M. Dynamics of Solvated Electrons in Clusters. Chem. Rev. 2012, 112, 5553-5577.

(13) Chen, X.; Bradforth, S. E. The Ultrafast Dynamics of Photodetachment. Annu. Rev. Phys. Chem. 2008, 59, 203-231.

(14) Bragg, A. E.; Verlet, J. R. R.; Kammrath, A.; Cheshnovsky, O.; Neumark, D. M. Electronic Relaxation Dynamics of Water Cluster Anions. J. Am. Chem. Soc. 2005, 127, 15283-15295.

(15) Griffin, G. B.; Young, R. M.; Ehrler, O. T.; Neumark, D. M. Electronic Relaxation Dynamics in Large Anionic Water Clusters: $\left(\mathrm{H}_{2} \mathrm{O}\right)_{\mathrm{N}}{ }^{-}$and $\left(\mathrm{D}_{2} \mathrm{O}\right)_{\mathrm{N}}{ }^{-}(\mathrm{N}=25-200)$. J. Chem. Phys. 2009, 131, 194302.

(16) Savolainen, J.; Uhlig, F.; Ahmed, S.; Hamm, P.; Jungwirth, P. Direct Observation of the Collapse of the Delocalized Excess Electron in Water. Nat. Chem. 2014, 6, 697-701.

(17) Coons, M. P.; You, Z. Q.; Herbert, J. M. The Hydrated Electron at the Surface of Neat Liquid Water Appears to Be Indistinguishable from the Bulk Species. J. Am. Chem. Soc. 2016, 138, 10879-10886.

(18) Uhlig, F.; Marsalek, O.; Jungwirth, P. Electron at the Surface of Water: Dehydrated or Not? J. Phys. Chem. Lett. 2013, 4, 338-343.

(19) Casey, J. R.; Schwartz, B. J.; Glover, W. J. Free Energies of Cavity and Noncavity Hydrated Electrons near the Instantaneous Air/ Water Interface. J. Phys. Chem. Lett. 2016, 7, 3192-3198.
(20) Borgis, D.; Rossky, P. J.; Turi, L. Electronic Excited State Lifetimes of Anionic Water Clusters: Dependence on Charge Solvation Motif. J. Phys. Chem. Lett. 2017, 8, 2304-2309.

(21) Herbert, J. M.; Coons, M. P. The Hydrated Electron. Annu. Rev. Phys. Chem. 2017, 68, 447-472.

(22) Stähler, J.; Deinert, J. C.; Wegkamp, D.; Hagen, S.; Wolf, M. Real-Time Measurement of the Vertical Binding Energy During the Birth of a Solvated Electron. J. Am. Chem. Soc. 2015, 137, 3520-3524.

(23) Sagar, D. M.; Bain, C. D.; Verlet, J. R. R. Hydrated Electrons at the Water/Air Interface. J. Am. Chem. Soc. 2010, 132, 6917-6919.

(24) Nowakowski, P. J.; Woods, D. A.; Verlet, J. R. R. Charge Transfer to Solvent Dynamics at the Ambient Water/Air Interface. J. Phys. Chem. Lett. 2016, 7, 4079-4085.

(25) Elkins, M. H.; Williams, H. L.; Neumark, D. M. Isotope Effect on Hydrated Electron Relaxation Dynamics Studied with TimeResolved Liquid Jet Photoelectron Spectroscopy. J. Chem. Phys. 2016, $144,184503$.

(26) Riley, J. W.; Wang, B. X.; Woodhouse, J. L.; Assmann, M.; Worth, G. A.; Fielding, H. H. Unravelling the Role of an Aqueous Environment on the Electronic Structure and Ionization of Phenol Using Photoelectron Spectroscopy. J. Phys. Chem. Lett. 2018, 9, 678682 .

(27) Coe, J. V.; Arnold, S. T.; Eaton, J. G.; Lee, G. H.; Bowen, K. H. Photoelectron Spectra of Hydrated Electron Clusters: Fitting Line Shapes and Grouping Isomers. J. Chem. Phys. 2006, 125, No. 014315.

(28) Ma, L.; Majer, K.; Chirot, F.; von Issendorff, B. Low Temperature Photoelectron Spectra of Water Cluster Anions. J. Chem. Phys. 2009, 131, 144303.

(29) Ehrler, O. T.; Neumark, D. M. Dynamics of Electron Solvation in Molecular Clusters. Acc. Chem. Res. 2009, 42, 769-777.

(30) Lietard, A.; Verlet, J. R. R. Selectivity in Electron Attachment to Water Clusters. J. Phys. Chem. Lett. 2019, 10, 1180-1184.

(31) Turi, L.; Rossky, P. J. Theoretical Studies of Spectroscopy and Dynamics of Hydrated Electrons. Chem. Rev. 2012, 112, 5641-5674.

(32) Kambhampati, P.; Son, D. H.; Kee, T. W.; Barbara, P. F. Solvation Dynamics of the Hydrated Electron Depends on Its Initial Degree of Electron Delocalization. J. Phys. Chem. A 2002, 106, 23742378.

(33) Lian, R.; Oulianov, D. A.; Shkrob, I. A.; Crowell, R. A. Geminate Recombination of Electrons Generated by above-the-Gap $(12.4 \mathrm{eV})$ Photoionization of Liquid Water. Chem. Phys. Lett. 2004, 398, 102-106.

(34) Kratz, S.; Torres-Alacan, J.; Urbanek, J.; Lindner, J.; Vöhringer, P. Geminate Recombination of Hydrated Electrons in Liquid-toSupercritical Water Studied by Ultrafast Time-Resolved Spectroscopy. Phys. Chem. Chem. Phys. 2010, 12, 12169-12176.

(35) Paik, D. H.; Lee, I.-R.; Yang, D.-S.; Baskin, J. S.; Zewail, A. H. Electrons in Finite-Sized Water Cavities: Hydration Dynamics Observed in Real Time. Science 2004, 306, 672-675.

(36) Wilhelm, J.; VandeVondele, J.; Rybkin, V. V. Dynamics of the Bulk Hydrated Electron from Many-Body Wave-Function Theory. Angew. Chem., Int. Ed. 2019, 58, 3890-3893.

(37) Luckhaus, D.; Yamamoto, Y. I.; Suzuki, T.; Signorell, R. Genuine Binding Energy of the Hydrated Electron. Sci. Adv. 2017, 3, No. e1603224.

(38) Karashima, S.; Yamamoto, Y.-I.; Suzuki, T. Ultrafast Internal Conversion and Solvation of Electrons in Water, Methanol, and Ethanol. J. Phys. Chem. Lett. 2019, 10, 4499-4504.

(39) LaForge, A. C.; Michiels, R.; Bohlen, M.; Callegari, C.; Clark, A.; von Conta, A.; Coreno, M.; Di Fraia, M.; Drabbels, M.; Huppert, M.; et al. Real-Time Dynamics of the Formation of Hydrated Electrons Upon Irradiation of Water Clusters with Extreme Ultraviolet Light. Phys. Rev. Lett. 2019, 122, 133001.

(40) Verlet, J. R. R.; Bragg, A. E.; Kammrath, A.; Cheshnovsky, O.; Neumark, D. M. Observation of Large Water-Cluster Anions with Surface-Bound Excess Electrons. Science 2005, 307, 93-96.

(41) Signorell, R.; Goldmann, M.; Yoder, B. L.; Bodi, A.; Chasovskikh, E.; Lang, L.; Luckhaus, D. Nanofocusing, Shadowing, 
and Electron Mean Free Path in the Photoemission from Aerosol Droplets. Chem. Phys. Lett. 2016, 658, 1-6.

(42) Michaud, M.; Wen, A.; Sanche, L. Cross Sections for LowEnergy (1-100 Ev) Electron Elastic and Inelastic Scattering in Amorphous Ice. Radiat. Res. 2003, 159, 3-22.

(43) Ottosson, N.; Faubel, M.; Bradforth, S. E.; Jungwirth, P.; Winter, B. Photoelectron Spectroscopy of Liquid Water and Aqueous Solution: Electron Effective Attenuation Lengths and Emission-Angle Anisotropy. J. Electron Spectrosc. Relat. Phenom. 2010, 177, 60-70.

(44) Thürmer, S.; Seidel, R.; Faubel, M.; Eberhardt, W.; Hemminger, J. C.; Bradforth, S. E.; Winter, B. Photoelectron Angular Distributions from Liquid Water: Effects of Electron Scattering. Phys. Rev. Lett. 2013, 111, 173005.

(45) Suzuki, Y.-I.; Nishizawa, K.; Kurahashi, N.; Suzuki, T. Effective Attenuation Length of an Electron in Liquid Water between 10 and 600 eV. Phys. Rev. E 2014, 90, No. 010302.

(46) Shinotsuka, H.; Da, B.; Tanuma, S.; Yoshikawa, H.; Powell, C. J.; Penn, D. R. Calculations of Electron Inelastic Mean Free Paths. XI. Data for Liquid Water for Energies from $50 \mathrm{eV}$ to $30 \mathrm{keV}$. Surf. Interface Anal. 2017, 49, 238-252.

(47) Olivieri, G.; Parry, K. M.; Powell, C. J.; Tobias, D. J.; Brown, M. A. Quantitative Interpretation of Molecular Dynamics Simulations for X-Ray Photoelectron Spectroscopy of Aqueous Solutions. J. Chem. Phys. 2016, 144, 154704.

(48) Zhang, C.; Andersson, T.; Förstel, M.; Mucke, M.; Arion, T.; Tchaplyguine, M.; Björneholm, O.; Hergenhahn, U. The Photoelectron Angular Distribution of Water Clusters. J. Chem. Phys. 2013, 138, 234306.

(49) Nishitani, J.; West, C. W.; Suzuki, T. Angle-Resolved Photoemission Spectroscopy of Liquid Water at $29.5 \mathrm{eV}$. Struct. Dyn. 2017, 4, No. 044014.

(50) Hartweg, S.; Yoder, B. L.; Garcia, G. A.; Nahon, L.; Signorell, R. Size-Resolved Photoelectron Anisotropy of Gas Phase Water Clusters and Predictions for Liquid Water. Phys. Rev. Lett. 2017, 118, 103402.

(51) Gartmann, T. E.; Hartweg, S.; Ban, L.; Chasovskikh, E.; Yoder, B. L.; Signorell, R. Electron Scattering in Large Water Clusters from Photoelectron Imaging with High Harmonic Radiation. Phys. Chem. Chem. Phys. 2018, 20, 16364-16371.

(52) Itikawa, Y.; Mason, N. Cross Sections for Electron Collisions with Water Molecules. J. Phys. Chem. Ref. Data 2005, 34, 1-22.

(53) Gartmann, T. E.; Yoder, B. L.; Chasovskikh, E.; Signorell, R. Lifetimes and Energetics of the First Electronically Excited States of $\mathrm{NaH}_{2} \mathrm{O}$ from Time-Resolved Photoelectron Imaging. Chem. Phys. Lett. 2017, 683, 515-520.

(54) Eppink, A.; Parker, D. H. Velocity Map Imaging of Ions and Electrons Using Electrostatic Lenses: Application in Photoelectron and Photofragment Ion Imaging of Molecular Oxygen. Rev. Sci. Instrum. 1997, 68, 3477-3484.

(55) Yoder, B. L.; West, A. H. C.; Schläppi, B.; Chasovskikh, E.; Signorell, R. A Velocity Map Imaging Photoelectron Spectrometer for the Study of Ultrafine Aerosols with a Table-Top VUV Laser and NaDoping for Particle Sizing Applied to Dimethyl Ether Condensation. J. Chem. Phys. 2013, 138, No. 044202.

(56) West, A. H. C.; Yoder, B. L.; Signorell, R. Size-Dependent Velocity Map Photoelectron Imaging of Nanosized Ammonia Aerosol Particles. J. Phys. Chem. A 2013, 117, 13326-13335.

(57) Signorell, R.; Yoder, B. L.; West, A. H. C.; Ferreiro, J. J.; Saak, C.-M. Angle-Resolved Valence Shell Photoelectron Spectroscopy of Neutral Nanosized Molecular Aggregates. Chem. Sci. 2014, 5, 12831295.

(58) West, A. H. C.; Yoder, B. L.; Luckhaus, D.; Signorell, R. Solvated Electrons in Clusters: Magic Numbers for the Photoelectron Anisotropy. J. Phys. Chem. A 2015, 119, 12376-12382.

(59) Ferray, M.; L'Huillier, A.; Li, X. F.; Lompre, L. A.; Mainfray, G.; Manus, C. Multiple-Harmonic Conversion of $1064 \mathrm{~nm}$ Radiation in Rare Gases. J. Phys. B: At., Mol. Opt. Phys. 1988, 21, L31-L35.

(60) Frassetto, F.; Cacho, C.; Froud, C. A.; Turcu, I. C. E.; Villoresi, P.; Bryan, W. A.; Springate, E.; Poletto, L. Single-Grating
Monochromator for Extreme-Ultraviolet Ultrashort Pulses. Opt. Express 2011, 19, 19169-19181.

(61) Frassetto, F.; Miotti, P.; Poletto, L. Grating Configurations for the Spectral Selection of Coherent Ultrashort Pulses in the ExtremeUltraviolet. Photonics 2014, 1, 442-454.

(62) Bobbert, C.; Schütte, S.; Steinbach, C.; Buck, U. Fragmentation and Reliable Size Distributions of Large Ammonia and Water Clusters. Eur. Phys. J. D 2002, 19, 183-192.

(63) Yoder, B. L.; Litman, J. H.; Forysinski, P. W.; Corbett, J. L.; Signorell, R. Sizer for Neutral Weakly Bound Ultrafine Aerosol Particles Based on Sodium Doping and Mass Spectrometric Detection. J. Phys. Chem. Lett. 2011, 2, 2623-2628.

(64) Coons, M. P.; Herbert, J. M. Quantum Chemistry in Arbitrary Dielectric Environments: Theory and Implementation of Nonequilibrium Poisson Boundary Conditions and Application to Compute Vertical Ionization Energies at the Air/Water Interface. J. Chem. Phys. 2018, 148, 222834.

(65) Zeuch, T.; Buck, U. Sodium Doped Hydrogen Bonded Clusters: Solvated Electrons and Size Selection. Chem. Phys. Lett. 2013, 579, 1-10.

(66) West, A. H. C.; Yoder, B. L.; Luckhaus, D.; Saak, C.-M.; Doppelbauer, M.; Signorell, R. Angle-Resolved Photoemission of Solvated Electrons in Sodium-Doped Clusters. J. Phys. Chem. Lett. 2015, 6, 1487-1492. 